\title{
Interpretation of trap-limited mobility in space-charge limited current in organic layers with exponential density of traps
}

Cite as: J. Appl. Phys. 110, 043705 (2011); https://doi.org/10.1063/1.3622615

Submitted: 24 March 2011 . Accepted: 29 June 2011 . Published Online: 17 August 2011

José M. Montero, and Juan Bisquert

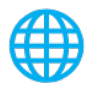

\section{ARTICLES YOU MAY BE INTERESTED IN}

Space-Charge-Limited Currents in Organic Crystals

Journal of Applied Physics 33, 205 (1962); https://doi.org/10.1063/1.1728487

Trap filled limit voltage (VTFL) and V2 law in space charge limited currents

Journal of Applied Physics 102, 094505 (2007); https://doi.org/10.1063/1.2802553

Space-charge limited conduction with traps in poly(phenylene vinylene) light emitting diodes Journal of Applied Physics 82, 6326 (1997); https://doi.org/10.1063/1.366523

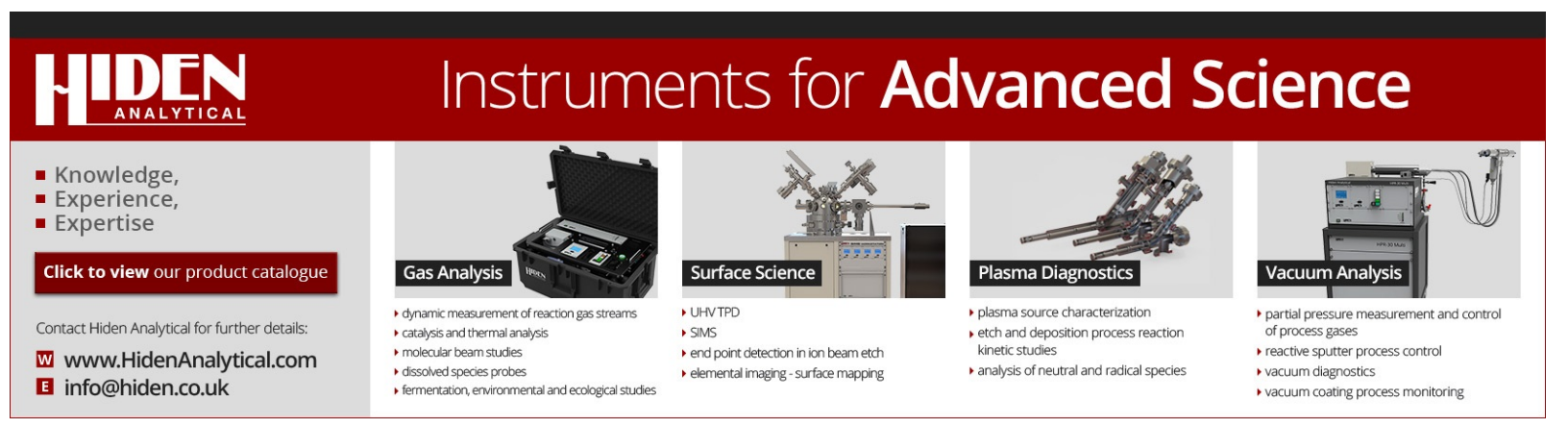




\title{
Interpretation of trap-limited mobility in space-charge limited current in organic layers with exponential density of traps
}

\author{
José M. Montero and Juan Bisquert ${ }^{a)}$ \\ Photovoltaics and Optoelectronic Devices Group, Departament de Física, Universitat Jaume I, 12071 \\ Castelló, Spain
}

(Received 24 March 2011; accepted 29 June 2011; published online 17 August 2011)

\begin{abstract}
Charge carrier transport in disordered organic semiconductors, performed in electronic devices such as optoelectronic and photovoltaic ones, is usually affected by an exponential distribution of localized states in the band-gap (traps) under space-charge limited current. In this paper, we provide a full analysis for the trap-controlled transport of the single-carrier device in the frequency domain. Trap-limited mobility is interpreted in terms of the classical multiple-trapping picture with one transport state and the trapping-detrapping dynamics of the exponential density of traps. This allows us to provide a suitable explanation of the usual experimental features of the mobility dependence on voltage as along with the capacitance spectra. (C) 2011 American Institute of Physics. [doi:10.1063/1.3622615]
\end{abstract}

\section{INTRODUCTION}

Over the last two decades, the application of light-emitting diodes (LEDs) to the technology of daily life has created an increasing interest, going beyond the traditional role of light indicators and displays to home appliances and general illumination. ${ }^{1,2}$ At the same time, an exhaustive technological research effort was triggered by the potential applications of the new ongoing generation of these devices based on organic semiconductors. ${ }^{3}$ Low-cost manufacturing processability and large area and flexible devices may be achieved by polymer-based organic light-emitting diodes. ${ }^{4,5}$ Nevertheless, the performance of organic electronic devices strongly depends upon the charge transport process carried out, therefore, further understanding of the physical behavior of such materials is needed. For instance, the description of charge transport in organic layers by space-charge limited current (SCLC) ${ }^{6-9}$ requires an interpretation of mobility by different semiempirical models (with field- or density-dependence), which is the crucial parameter governing the transport in the bulk. ${ }^{10}$ Recently, ${ }^{11}$ we have treated the relationship between the different models and in this paper we provide a full analysis of the interpretation of the capacitance and conductance spectra, along with a comparison with the observed experimental features.

In the 1990s, field-dependent mobility models were proposed by Bässler, as a result of assuming hopping transport in a Gaussian density of states (DOS). ${ }^{12}$ Field-dependent mobility in organic layers, such as in sandwiched films composed of either poly(p-phenylene vinylene) (PPV) derivatives or aluminum hydroxyquinoline (Alq3), became widely accepted. However, Tanase et al. presented a comparison of mobility values for two solution-processed organic polymers: poly(2-methoxy-5-(3',7'-dimethyloctyloxy)-p-phenylene vinylene) $\left(\mathrm{OC}_{1} \mathrm{C}_{10}\right.$-PPV) and poly(3-hexyl thiophene) (P3HT), performed in two different configurations, i.e., field-effect transistors and hole-only diodes. ${ }^{13}$ The first

a)Electronic mail: bisquert@fca.uji.es. structure displayed mobility results up to three orders of magnitude higher than the latter configuration. These observations endorsed the density-dependent mobility model, proposed by Vissenberg and Matters in amorphous organic transistors, that stems from hopping percolation in an exponential DOS. ${ }^{14}$ As demonstrated by Arkhipov et al., hopping transport in disordered materials can be reduced to a trapcontrolled transport composed of an effective transport level and a broad distribution of localized states (traps) that only retain mobile charges. ${ }^{15}$ Currently, several authors are considering this framework: transport via an extended state under the influence of an exponential density of traps. ${ }^{11,16-18}$ In the present paper, we implement this assumption in SCLC to analyze its implications for charge carrier mobility.

From an experimental point of view, the determination of mobility is commonly given through the study of transit times (i.e., time needed for carriers to cross the sample electrode-to-electrode) in the wide range of the methods available in the literature: time-of-flight, transient electroluminescence, dark injection, and impedance spectroscopy (IS), among others. ${ }^{19,20}$ The IS technique will focus our computational calculations to provide physical insights on the experimental measurements of capacitance spectra. As demonstrated in an earlier theoretical work of a single-carrier device in SCLC with only a single trap, ${ }^{21}$ there is a strong correlation between the shape of the capacitance spectra and the nature of traps lying in the band-gap. In particular, a classification of them was established using its energy depth and its dynamic activity to capture and release charge carriers (i.e., shallow: fast and slow, and deep traps). Meanwhile fast-shallow traps were responsible for the delay of transit times (i.e., shifted capacitance step-ups), slow-shallow traps were for low-frequency capacitance increases. The aim of this paper is to extend these ideas to a wider set of traps, from a single to an exponential density in the band-gap, ${ }^{11}$ in order to further test the theoretical framework with experimental capacitance data: a single-carrier device composed of $\mathrm{N}, \mathrm{N}^{\prime}$-diphenyl-N,N'-bis(1-naphtylphenyl)-1,1'-biphenyl-4,4'diamine $(\alpha-\mathrm{NPD})$. 
This paper is structured as follows: first, we discuss the exponential-density-trap model; second, the results and discussion section; and finally, the conclusions.

\section{THE EXPONENTIAL-DENSITY-TRAP MODEL}

The SCLC regime for electron transport in disordered organic semiconductors comprises two classes of energy states: one transport level with density, $n_{c}$, that drifts in the electric field, $F$, and an exponential distribution of localized states with density, $n_{t}$, that corresponds to the immobilized trapped charge. The mathematical description is well-known in the literature and entails the continuity equation, the drift-current equation, and the Poisson equation, respectively, ${ }^{22}$

$$
\begin{gathered}
\frac{d J}{d x}=0, \\
J=q \mu_{0 n} n_{c} F+\varepsilon_{r} \varepsilon_{0} \frac{\partial F}{\partial t}, \\
\frac{d F}{d x}=\frac{q}{\varepsilon_{r} \varepsilon_{0}}\left(n_{c}+n_{t}\right) .
\end{gathered}
$$

Furthermore, the trap dynamics equation is considered for every energy level, $E_{t}$, in the exponential distribution of traps with occupancy, $f_{t}\left(E_{t}\right)$, along the band-gap as, ${ }^{23}$

$$
\frac{\partial f_{t}}{\partial t}=c n_{c}\left[1-f_{t}\right]-e f_{t} .
$$

Here, $q$ is the elementary charge, $\mu_{0 n}$ is the trap-free mobility, $\varepsilon_{r} \varepsilon_{0}$ is the dielectric constant, and $c$ and $e$ are the coefficients for electron capture and release, respectively. The potential can be calculated by integrating the electric field along the thickness, $L$,

$$
V=\int_{0}^{L} F d x .
$$

In fact, the same system of equations stands for the hole transport just by swapping the spatial and energy scales origins at once. The population of the extended states at the energy level, $E_{c}$, for a non-degenerate semiconductor, relates to the Fermi level, $E_{F}$, as

$$
n_{c}\left(E_{F}\right)=N_{c} e^{\left(E_{F}-E_{c}\right) / k_{B} T},
$$

where $N_{c}$ is an effective density of states in the transport level.

The trapped population is the overall density of charge carriers located by the exponentially distributed traps along the band-gap,

$$
\begin{gathered}
n_{t}\left(E_{F}\right)=\int_{E_{V}}^{E_{c}} g_{t}\left(E_{t}\right) f_{t}\left(E_{t}, E_{F}\right) d E_{t}, \\
g_{t}\left(E_{t}\right)=\frac{N_{t}}{k_{B} T_{t}} e^{\frac{E_{t}-E_{c}}{k_{B} T_{t}}}
\end{gathered}
$$

where $N_{t}$ is an effective density of traps and $T_{t}$ is the characteristic trap temperature.
Assuming that every trap energy level, $E_{t}$, reaches equilibrium with the extended state (with the same Fermi level), the trap occupancy is given by,

$$
f_{t}\left(E_{t}, E_{F}\right)=\frac{1}{1+e^{\left(E_{t}-E_{F}\right) / k_{B} T}} .
$$

In steady state, Eq. (4) yields,

$$
f_{t}=\frac{1}{1+e /\left(c n_{c}\right)} .
$$

Therefore, the detailed balance condition provides the following relationship for the trap emission and capture coefficients, Eqs. (6) and (9), into Eq. (10) yields,

$$
e=c N_{c} e^{\left(E_{t}-E_{c}\right) / k_{B} T} .
$$

Let us denote the steady-state by $\bar{x}$ and a small perturbation by $\hat{x}$ applied at a certain angular frequency, $\omega$. Hence, every electrical variable can be expressed as $x=\bar{x}+\hat{x}$ to linearize the whole system of equations up to the first order. ${ }^{24}$ As shown in Ref. 25, by solving Eq. (4) for a small perturbation, we obtain,

$$
\hat{f}_{t}\left(E_{t}\right)=\frac{1}{\bar{n}_{c}} \frac{\bar{f}_{t}\left(1-\bar{f}_{t}\right)}{1+i \omega / \omega_{t}} \hat{n}_{c} .
$$

This term gives the contribution to the spectra of the capacitance and conductance of every trap level. Every trap frequency is defined as

$$
\omega_{t}\left(E_{t}\right)=\frac{e}{1-\bar{f}_{t}} .
$$

This is the maximum frequency that the trap is acting as such, since at higher frequencies the trap cannot follow the ac perturbation. ${ }^{21}$ Inserting Eq. (11) into Eq. (13), we find the dependence of $\omega_{t}$ on the trap energy and occupation, as

$$
\omega_{t}=\frac{c N_{c} e^{\left(E_{t}-E_{c}\right) / k_{B} T}}{1-\bar{f}_{t}} .
$$

It should be noted that in the SCLC regime, $\bar{f}_{t}$ is positiondependent along the organic layer. The impedance is defined as the quotient of the potential to the current density,

$$
Z(\omega)=\frac{\hat{V}(\omega)}{\hat{J}(\omega)} .
$$

The quantity, $\hat{V}(\omega)$, is determined by the spatial integration of $\hat{F}(\omega)$ from the solution of the preceding model. The boundary conditions at the injecting contact used to solve the electrical variables along the thickness in dc and ac conditions are, ${ }^{26,27}$

$$
\bar{n}_{c}(x=0)=N_{c} \quad \text { and } \quad \hat{F}(x=0)=0 .
$$

The capacitance and conductance are defined as follows:

$$
C(\omega)=\operatorname{Re}\left[\frac{1}{i \omega Z(\omega)}\right],
$$




$$
g(\omega)=\operatorname{Re}\left[\frac{1}{Z(\omega)}\right] .
$$

The dielectric capacitance of the organic layer is denoted as $C_{g}$.

The present physical model leads to a first-order differential equation. The numerical algorithm used to achieve the solution is based on a double discretization; one for the thickness $L$ (i.e., spatial coordinate $x$ ), and the other one for the energy bandgap (i.e., trap energy $E_{t}$ ). On the one hand, the steady-state is solved from Eqs. (1)-(3) and Eqs. (5)-(9) by pinning the mobile carriers as the effective density of states at the injecting contact in Eq. (16). On the other hand, the time-dependent regime stems from the application of an additional small ac voltage over the dc one, therefore, the inclusion of the time-dependent trapping action is required, as shown in Eq. (4) and Eqs. (10)-(14). The ac boundary condition is set to zero electric field at the injecting contact [Eq. (16)] and the frequency-response solution [Eq. (15)] is obtained.

\section{RESULTS AND DISCUSSION}

\section{A. Theoretical framework}

\section{Steady-state characteristics of organic layers with an exponential density of traps}

Experimental measurements of $J-V$ curves are usually analyzed to interpret the performance behavior and charge transport of a wide range of electronic devices, such as organic light-emitting diodes, ${ }^{28}$ transitors $^{13}$ and solar cells. ${ }^{29}$ In our case, for single-carrier organic layers, we calculate the steady-state solution from the preceding model and the results are displayed in Fig. 1. Input data are shown in Table I. At low voltages, most of the ohmically injected charges are trapped $\left(n_{t} \gg n\right.$, in the Poisson equation) and the representation approaches the formula, ${ }^{16,17}$

$$
J=e \mu_{0 n} N_{c}\left(\frac{\varepsilon}{e N_{t}}\right)^{l}\left(\frac{l}{l+1}\right)^{l}\left(\frac{2 l+1}{l+1}\right)^{l+1} \frac{V^{l+1}}{L^{2 l+1}},
$$

whereas at high voltages, $n_{t} \ll n$, the Mott-Gourney square law modified by shallow traps is followed,

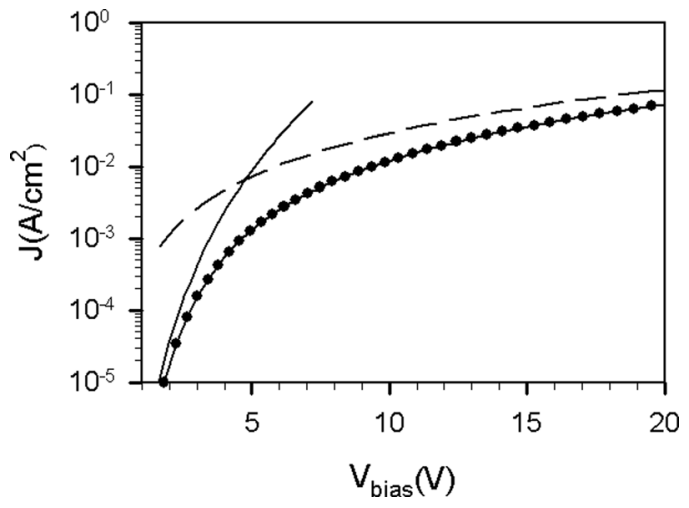

FIG. 1. Simulations of current-density-voltage characteristics (line and scatterplot) pictured together with the analytical formulas: Eq. (19) (solid line) and Child's law, Eq. (20) with $\theta=1$ (dashed line).
TABLE I. Material parameters implemented for simulations.

\begin{tabular}{ll}
\hline \hline Parameter & Value \\
\hline Thickness, $L$ & $80 \mathrm{~nm}$ \\
Transport effective density of states, $N_{c}$ & $10^{19} \mathrm{~cm}^{-3}$ \\
Relative dielectric constant, $\varepsilon_{r}$ & 3 \\
Trap-free mobility, $\mu_{0 n}$ & $5 \times 10^{-7} \mathrm{~cm}^{2} /(\mathrm{Vs})$ \\
Temperature, $T$ & $300 \mathrm{~K}$ \\
Band-gap, $E_{c}-E_{v}$ & $2.4 \mathrm{eV}$ \\
Trap effective density of states, $N_{t}$ & $5 \times 10^{17} \mathrm{~cm}^{-3}$ \\
Characteristic trap temperature, $T_{t}$ & $1500 \mathrm{~K}$ \\
Trapping capture coefficient, $c$ & $7 \times 10^{-14} \mathrm{~cm}^{3} / \mathrm{s}$ \\
\hline \hline
\end{tabular}

$$
J=\frac{9}{8} \varepsilon \theta \mu_{0 n} \frac{V^{2}}{L^{3}},
$$

where $\theta^{-1}\left(=1+\left\langle n_{t}\right\rangle /\left\langle n_{c}\right\rangle\right)$ is a carrier-density dependent factor of trapped and free charge located by the shallow traps. ${ }^{30}$ It should be remarked that trap dynamics [Eq. (4)] have not been included in the present calculations and the mobility parameter is thereby defined as $\mu_{0 n}$, which is independent of voltage.

\section{Impedance response of organic layers with an exponential density of traps}

Experimental measurements of the capacitance spectra extracted from the impedance response [Eq. (17)] are considered a powerful tool to determine the charge transport parameters such as carrier mobility for holes and electrons. ${ }^{19,31,32}$

The model described in Sec. II was already analytically solved by Dascalu with the approximation of $J \propto V^{2}$ and two suggested trapping coefficients independent of occupation $(\delta$ and $\Psi$ ). However, this approach does not take into account the voltage dependence of mobility if extended to any voltage range. ${ }^{3-35}$ In contrast, our computational results of the capacitance with the exponential density of traps have no restrictions and cover the whole model previously exposed. Figure 2(a) shows capacitance calculations with the material parameters of Table I. Deviation from the wellknown trap-free spectrum, i.e., a step up from $0.75 C_{g}$ to $C_{g}$ at a certain frequency, ${ }^{8,36}$ is displayed as a reference. Lowfrequency capacitance exhibits an increased value far more than the traditional $0.75 C_{g}$ and this behavior is attributed to the slow-shallow traps within the distribution that cannot achieve the quasi-equilibrium with the transport level. At higher frequencies approaching the transit time, the trapping-detrapping dynamics of fast-shallow traps intersect with the transit of charge carriers from the injecting to the collecting contacts, thus causing a time delay, as shown by peaks (arrows) in Fig. 2(b). Trap-limited mobility is interpreted in terms of this trap-controlled transport. Both roles of shallow traps (slow and fast) now occur at once with the exponential density of traps, unlike in our previous work of a single-trap. ${ }^{21}$ The deep traps contribution is also slightly observed in the first peak of the capacitance. As a minor comment, other capacitance simulations with a slower 


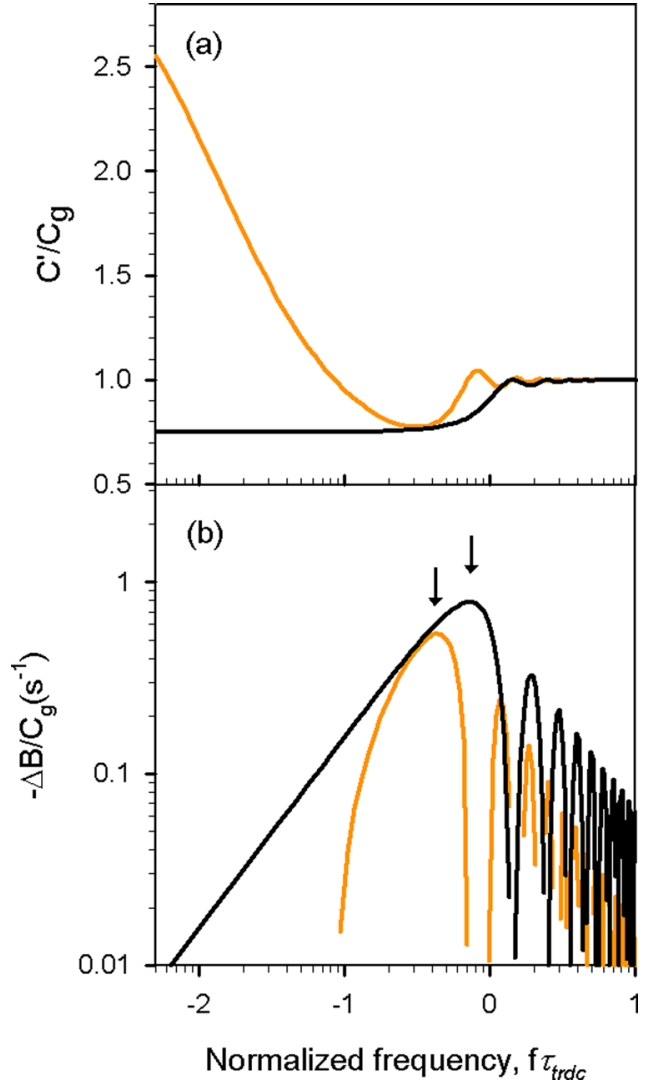

FIG. 2. (Color online) Model simulations at $3 \mathrm{~V}$ of (a) capacitance, and (b) differential susceptance spectra [see Eq. (24)], with an exponential density of traps (orange dashed line) in comparison to the trap-free spectrum (black solid line). Frequencies are normalized to transit time, $\tau_{t r d c}$, [see Eq. (26)].

capture trap rate yielded slightly lower values than $0.75 C_{g}$ in the vicinity of the transit time frequency.

Since we are dealing with an inhomogeneous system, the Fermi level and occupation vary along the thickness [Fig. 3(a)], especially abruptly close to the injection region and with a smoother functional dependence in the wide range of the sample thicknesses. Therefore, an average Fermi level $\left\langle E_{F}\right\rangle$ can be given as an appropriate approximation to simplify the theoretical analysis. Thus, the average occupancy becomes,

$$
\left\langle\bar{f}\left(E_{t}, E_{F}\right)\right\rangle \approx \bar{f}\left(E_{t},\left\langle E_{F}\right\rangle\right),
$$

and Fig. 3(b) shows the average occupied density of traps and the empty ones under the preceding approach. Presumably, the most shallow and empty traps will behave as fast, whereas the less shallow and full ones will be slow [see Eq. (14)]. Nevertheless, a further approach to the trapping rates along the energy distribution in the band-gap is of prime importance to disentangle the different roles of every trap energy state in the contribution to the capacitance spectra (see Fig. 4). The capture rate, $c$, is defined as energy independent with a higher value than the normalized emission rate, $e / N_{c}$, given in Eq. (11). However, the definitive discriminant parameters used to classify whether the shallow energy states are purely fast or not, are: the capture rate, $c$, and the critical capture coefficient, $c_{c}$, defined as,

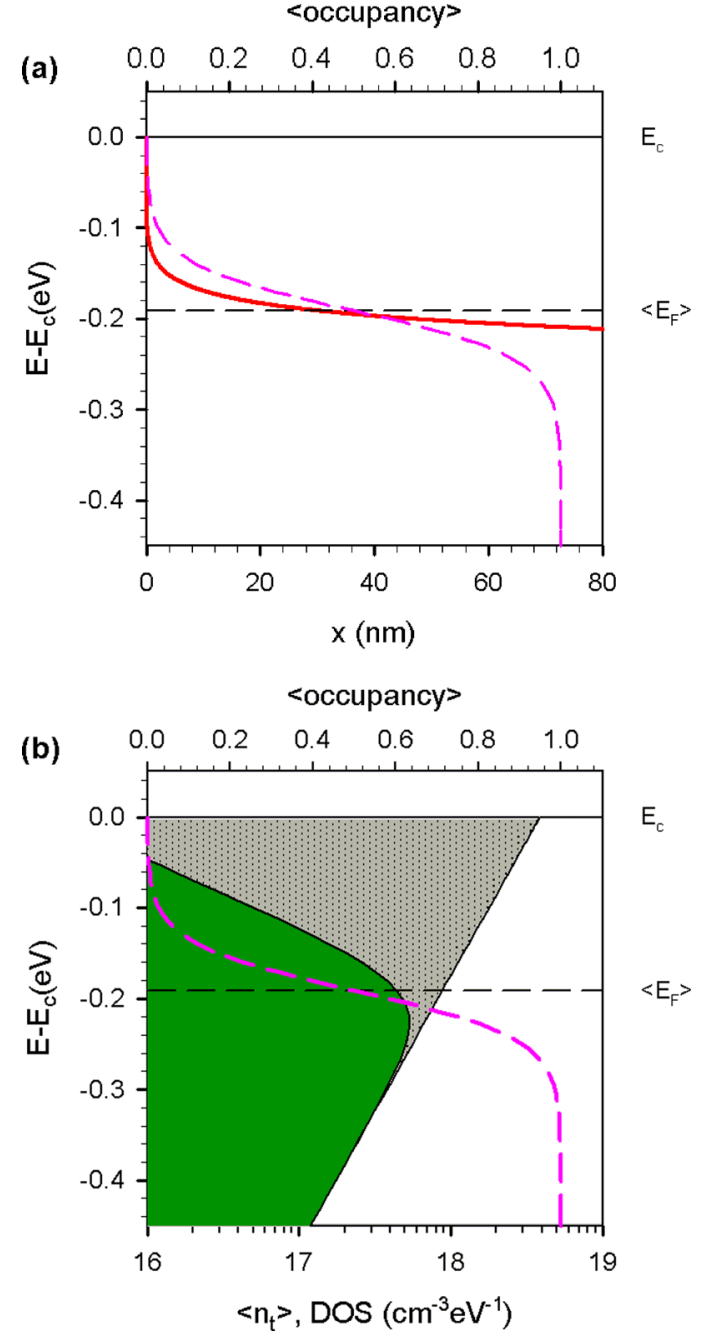

FIG. 3. (Color online) (a) Simulations at $3 \mathrm{~V}$ of the Fermi level along the thickness (solid line) and average occupancy (dashed line) that stems from the average Fermi level, $\left\langle E_{F}\right\rangle$. (b) Colored area is displayed for the average occupied trap density of states (DOS) whereas shadowed area corresponds to the empty trapping density of states. Average occupancy is also printed (pink dashed line) together with average Fermi level, $\left\langle E_{F}\right\rangle$, and transport energy level, $E_{c}$, as references.

$$
c_{c}\left(E_{t}\right) \approx \frac{3 \pi \mu_{0 n} V}{2 L^{2}} \frac{1-\bar{f}_{t}\left(E_{t},\left\langle E_{F}\right\rangle\right)}{N_{c} e^{\left(E_{t}-E_{c}\right) / k_{B} T}},
$$

and its discussion was also published in Ref. 21. If $c$ is larger than $c_{c}$ along a certain energy interval in the band-gap, these shallow energetic levels behave as fast-shallow traps resulting in a larger transit time and thereby, in a trap-limited mobility. This energy region below the transport level $\left(E_{c} \geq E_{t} \geq E_{L}\right)$ can be calculated as, $c_{c}\left(E_{L}\right) \cong c$, where $E_{L}$ is interpreted as the lowest energy level acting as purely fast,

$$
E_{L}=k_{B} T L n\left[\left(\frac{3 \pi \mu_{0} V}{c N_{c} L^{2}}\right) \exp \left[\frac{\left(E_{c}-\left\langle E_{F}\right\rangle\right)}{k_{B} T}\right]-1\right]+\left\langle E_{F}\right\rangle,
$$

which is dependent upon the voltage, $V$, dropped along the organic layer in space-charge, the Fermi level $\left\langle E_{F}\right\rangle$, and the device specifications. For shallow levels below $E_{L}$ 


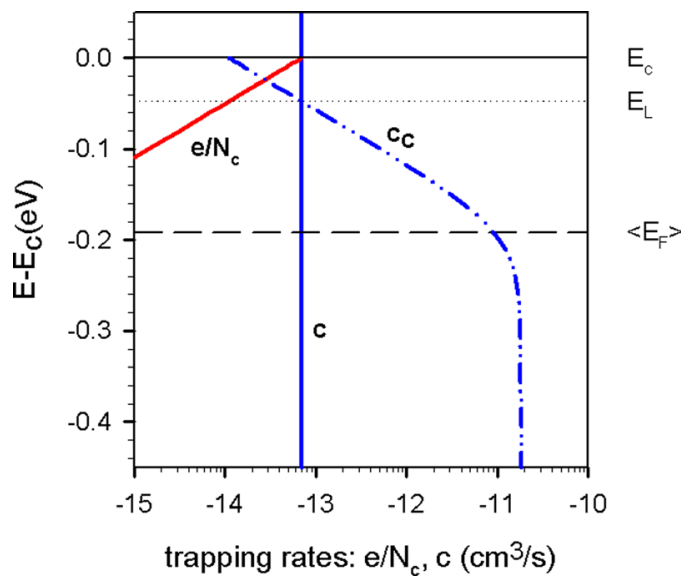

FIG. 4. (Color online) Trapping coefficients are displayed for the $3 \mathrm{~V}$ model simulation: normalized emission rate $\left(e / N_{c}\right)$ and trapping rate $(c)$ are represented by colored solid lines. Critical capture coefficient is also given by a dash-dotted line. Reference energy levels are: transport $E_{c}$, limit $E_{L}$, and average Fermi $\left\langle E_{F}\right\rangle$ levels.

$\left(E_{L}>E_{t}>\left\langle E_{F}\right\rangle\right)$, the trap dynamics start to gradually change from fast to slow, as far as the deeper states with which we are dealing. That makes it difficult to quantify the reduced factor, $\theta$, that commonly relates the trap-limited mobility to the trap-free one. ${ }^{30}$

In order to experimentally test the model,${ }^{37}$ capacitance spectra are generated at different voltages in Fig. 5. By increasing the voltage dropped in the space-charge, the Fermi level moves up toward the transport level, covering more trapping states in the band-gap. As a consequence, the range of the energy distribution acting as purely fast $\left(E_{c} \geq E_{t} \geq E_{L}\right)$ becomes narrowed since $E_{L}$ [Eq. (23)] also moves up. This indicates that there are less unoccupied trapping sites that can trap and release charges quickly while they are drifted electrode-to-electrode by the local electric field. In other words, trap limitation of mobility becomes lower the more voltage is applied since less fast-shallow traps are acting as such. As regards the low-frequency behavior with the voltage, the same reasoning can be given, since the energy region $\left(E_{L}>E_{t}>\left\langle E_{F}\right\rangle\right)$ containing the slowshallow traps, also becomes more reduced. Thus, less lowfrequency increase is expected the more voltage is applied.

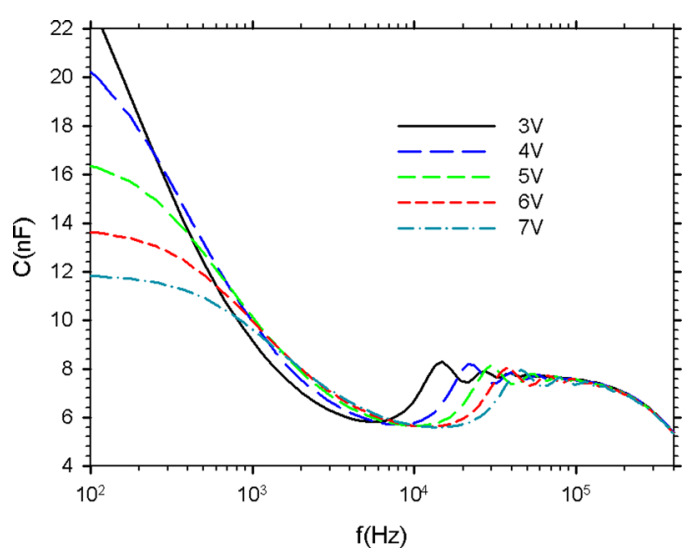

FIG. 5. (Color online) Model representation of capacitance spectra at voltages ranging from 3 to $7 \mathrm{~V}$. Simulation parameters are shown in Table I.

\section{B. Experimental analysis}

Let us now apply the model and theoretical framework to interpret the capacitance spectra of the hole-only device based on the $\alpha$-NPD of Nguyen $e t a l .{ }^{38}$ In that publication, the authors model the data by considering drift-diffusion transport with a Gaussian density of traps and field-dependent mobility, however, the low-frequency capacitance exhibits a rather sharp behavior in contrast to the experiments. This feature may be better described by an exponential distribution of traps that would result in a smoother capacitance variation at low-frequency.

We show the experimental capacitance spectra at different voltages of a thick hole-only device retrieved from Ref. 38 in Fig. 6 . The capacitance behavior and shape agrees well with our model at every frequency range, however, not the magnitudes, mainly due to a noticeable difference of thickness, $L$, from simulations (Fig. 5), in contrast to the experimental data shown in Fig. 6. The low-frequency (LF) capacitance part displays an increase, which is directly modulated by the trapping distribution, and concretely by the slow-shallow traps. The LF capacitance increase is more noticeable the less the voltage is applied, i.e., for the lower set of voltages $(3-5 \mathrm{~V})$ more than for the higher ones $(9-10 \mathrm{~V})$, as expected. The intermediate frequency (IF) range is characterized by the presence of a minimum value of the capacitance spectra and its position shifts depending on the voltage. Our model predicts all of the minima achieving the value of 0.75 of the geometrical capacitance, $C_{g}$, of the organic layer, however, the experimental data lies more over this limit the less the voltage is applied. This feature seems to be better described by Nguyen's model. In the IF region it is believed that the average transit time of the carriers, $\tau_{t r}$, can be extracted by means of the IS technique with the representation of the susceptance $(=\operatorname{Im}(Y))$, concretely, the negative differential susceptance,

$$
-\Delta B(\omega)=-\omega\left(C(\omega)-C_{g}\right) .
$$

The position of the maxima define the peaks corresponding to ac transit times at different voltages, therefore, ${ }^{39}$

$$
\tau_{\text {trac }} \approx 0.72 \cdot f_{\max }^{-1},
$$

and the mobility values can be extracted by using the dc transit time expression,

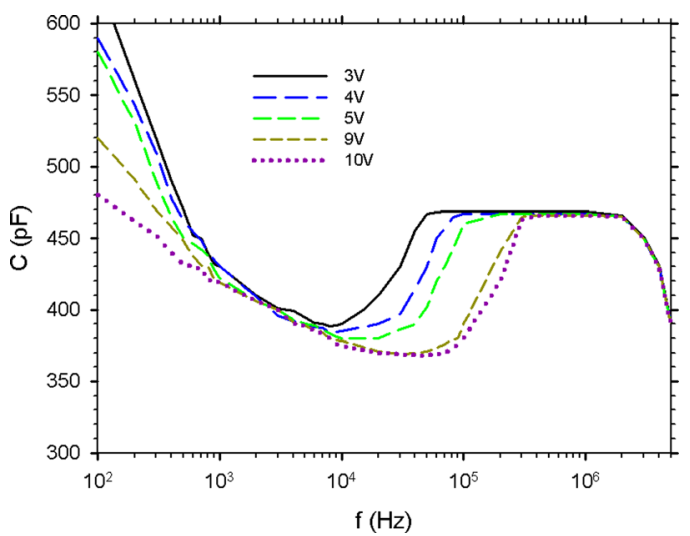

FIG. 6. (Color online) Experimental capacitance spectra of a hole-only $\alpha$-NPD device at different voltages. Contact area, $A$, is $0.235 \mathrm{~cm}^{2}$. 


$$
\tau_{\text {trdc }}=\frac{4}{3} \frac{L^{2}}{\mu\left(V_{\text {bias }}-V_{b i}\right)} .
$$

Inserting Eq. (25) into Eq. (26), it holds,

$$
\mu=\frac{4}{3} \frac{L^{2} f_{\max }}{0.72 \cdot\left(V_{\text {bias }}-V_{b i}\right)} .
$$

Calculations of mobility yielded mobility enhancement the more the voltage is applied in the bulk. In particular, the fitting to the classical field-dependent mobility expression,

$$
\mu=\mu_{0} \exp (\gamma \sqrt{F})
$$

with the approximation of $\sqrt{F} \approx \sqrt{V / L}$, provided experimental mobility values of $\mu_{0}=2.9 \times 10^{-4} \mathrm{~cm}^{2} /(\mathrm{Vs})$ and $\gamma=2.6 \times 10^{-3}(\mathrm{~cm} / \mathrm{V})^{1 / 2}$. This mobility dependence upon voltage, measured by means of IS, is interpreted in the present paper as a trap-limited mobility governed by the dynamics of the fast-shallow traps in the band-gap. The field-dependent mobility is induced by the reduction of the trapping action as far as more voltage covers more trapping sites in the exponential distribution of localized-states. As regards the high frequency range $(\mathrm{HF})$; it is composed of two different parts: a relatively wide plateau lying at the capacitance value of $C_{g}$, and a sharp decrease from a frequency cutoff onwards. The latter behavior of the HF is dominated by the series resistance of the whole device structure causing the capacitance drop.

In summary, the shape of the capacitance spectra with an exponential density of traps is strongly determined by the bias-voltage, providing: (1) a deviation of transit times translated into a field-dependent mobility, and (2) a low-frequency capacitance increase over the traditional $0.75 C_{g}$ for trap-free materials.

\section{CONCLUSIONS}

We have corroborated the theoretical framework of the multiple-trapping picture in organic layers that comprises an exponential density of trapping states under SCLC. The analysis of the capacitance spectra facilitates the interpretation of the voltage dependence of the trap-limited mobility and the low-frequency capacitance behavior. The presence of pure fast-shallow traps determines the limitation of the charge transport mobility, whereas slow-shallow traps cause the low-frequency capacitance to increase. Both features are modulated by two respective voltage-dependent energy regions in the band-gap.

\section{ACKNOWLEDGMENTS}

We are thankful for financial support from MEC of Spain under program Consolider-Ingenio 2010 (Project
HOPE CSD2007-00007), and Generalitat Valenciana under Project PROMETEO/2009/058.

${ }^{1}$ K. Meerholz, Nature (London) 437, 327 (2005).

${ }^{2}$ T. P. Nguyen, Phys. Status Solidi A 205, 162 (2008).

${ }^{3}$ R. H. Friend, R. W. Gymer, A. B. Holmes, J. H. Burroughes, R. N. Marks, C. Taliani, D. D. C. Bradley, D. A. Dos Santos, J. L. Brédas, M. Lögdlund, and W. R. Salaneck, Nature (London) 397, 121 (1999).

${ }^{4}$ J. H. Burroughes, D. D. C. Bradley, A. R. Brown, R. N. Marks, K. MacKay, R. H. Friend, P. L. Burn, and A. B. Holmes, Nature (London) 347, 539 (1990).

${ }^{5}$ S. R. Forrest, Nature (London) 428, 911 (2004).

${ }^{6}$ N. F. Mott and R. W. Gurney, Electronic Processes in Ionic Crystals, 2nd ed. (Oxford University Press, London, 1940).

${ }^{7}$ G. T. Wright, Solid-State Electron. 2, 165 (1961).

${ }^{8}$ J. Shao and G. T. Wright, Solid-State Electron. 3, 291 (1961).

${ }^{9}$ J. Lindmayer and A. Slobodskoy, Solid-State Electron. 6, 495 (1963).

${ }^{10}$ M. Jaiswal and R. Menon, Polym. Int. 55, 1371 (2006).

${ }^{11} \mathrm{~J}$. M. Montero and J. Bisquert, Solid-State Electron. 55, 1-4 (2011).

${ }^{12}$ H. Bässler, Phys. Status Solidi B 175, 15 (1993).

${ }^{13}$ C. Tanase, E. J. Meijer, P. W. M. Blom, and D. M. Leeuw, Phys. Rev. Lett. 91, 216601 (2003).

${ }^{14}$ M. C. J. M. Vissenberg and M. Matters, Phys. Rev. B 57, 12964 (1998).

${ }^{15}$ V. I. Arkhipov, E. V. Emelianova, and G. J. Adriaenssens, Phys. Rev. B 64, 125125 (2001).

${ }^{16}$ B. Ramachandhran, H. G. A. Huizing, and R. Coehoorn, Phys. Rev. B 73, 233306 (2006).

${ }^{17}$ M. M. Mandoc, B. de Boer, and P. W. M. Blom, Phys. Rev. B 73, 155205 (2006).

${ }^{18}$ M. M. Mandoc, B. de Boer, G. Paasch, and P. W. M. Blom, Phys. Rev. B 75, 193202 (2007).

${ }^{19}$ F. So, B. Krummacher, D. Poplavskyy, S. A. Choulis, and V. Choong, J. Appl. Phys. 102, 091101 (2007).

${ }^{20}$ S. Shirota and H. Kageyama, Chem. Rev. 107, 953 (2007).

${ }^{21}$ J. M. Montero, J. Bisquert, G. Garcia-Belmonte, E. M. Barea, and H. J. Bolink, Org. Electron. 10, 305 (2009).

${ }^{22}$ A. Van der Ziel, "Solid State Physical Electronics" (Prentice-Hall International, London, 1972).

${ }^{23}$ J. Bisquert and V. S. Vikhrenko, Electrochim. Acta 47, 3977 (2002).

${ }^{24}$ W. Brütting and S. Berleb, Phys. Rev. Lett. 89, 286601 (2002).

${ }^{25}$ J. Bisquert, Phys. Rev. B 77, 235203 (2008).

${ }^{26}$ P. W. M. Blom, M. J. M. de Jong, and M. G. van Munster, Phys. Rev. B 55, R656 (1997).

${ }^{27}$ M. A. Lampert and P. Mark, Current Injection in Solids, (Academic, New York, 1970), Vol. 10.

${ }^{28}$ G. Garcia-Belmonte, J. M. Montero, Y. Ayyad-Limonge, E. M. Barea, J. Bisquert, and H. Bolink, Curr. Appl. Phys. 9, 414 (2009).

${ }^{29}$ I. Mora-Seró, S. Giménez, T. Moehl, F. Fabregat-Santiago, T. LanaVillarreal, R. Gómez, and J. Bisquert, Nanotechnology 19, 424007 (2008).

${ }^{30}$ A. Rose, Concepts in Photoconductivity and Allied Problems (John Wiley \& Sons, New York, 1963)

${ }^{31}$ H. C. F. Martens, J. N. Huiberts, and P. W. M. Blom, Appl. Phys. Lett. 77, $1852(2000)$

${ }^{32}$ M. Schmeits, J. Appl. Phys. 101, 084508 (2007).

${ }^{33}$ D. Dascalu, Int. J. Electron. 21, 183 (1966).

${ }^{34}$ D. Dascalu, Solid-State Electron. 9, 1020 (1966).

${ }^{35}$ T. Okachi, T. Nagase, T. Kobayashi, and H. Naito, Appl. Phys. Lett. 94, 043301 (2009).

${ }^{36}$ S. W. Tsang, S. K. So, and J. B. Xu, J. Appl. Phys. 99, 013706 (2006).

${ }^{37}$ J. Bisquert, Phys. Chem. Chem. Phys. 10, 3175 (2008).

${ }^{38}$ N. D. Nguyen and M. Schmeits, Phys. Rev. B 75, 075307 (2007).

${ }^{39}$ J. M. Montero, J. Bisquert, G. Garcia-Belmonte, H. Bolink, and E. M. Barea, Phys. Status Solidi A 204, 2402 (2007). 